\title{
Consulta de enfermagem pré-procedimento de cateterismo cardíaco: avaliação da satisfação do paciente
}

\author{
Nursing consultation prepares cardiac catheterism procedure: assessment of patient satisfaction \\ Consulta de enfermería antes del procedimiento de cateterismo cardíaco: evaluación de la satisfacción \\ del paciente
}

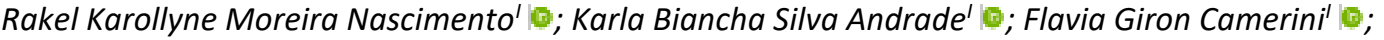 \\ Andrezza Serpa Franco' ${ }^{\circ}$; Ana Lúcia Cascardo Marins' ${ }^{\bullet}$; Camila Benicá de Oliveira Carvalho Naves'
}

'Universidade do Estado do Rio de Janeiro, Rio de Janeiro, RJ, Brasil

\begin{abstract}
RESUMO
Objetivo: avaliar a satisfação dos pacientes em relação à consulta de enfermagem prévia ao procedimento de cateterismo cardíaco. Método: estudo quantitativo, descritivo, realizado na hemodinâmica de um hospital universitário no estado do Rio de Janeiro, com a participação de 38 pacientes que responderam ao questionário da pesquisa, aprovado pelo Comitê de Ética em Pesquisa. Resultados: predominou o sexo masculino, 20 (53\%), com faixa etária acima dos 60 anos, 23 (60\%). A hipertensão arterial foi a comorbidade mais prevalente, 33 (87\%), tendo a angina como motivo principal, 22 (58\%). A satisfação do usuário com a consulta de enfermagem foi comprovada por meio do alto grau de concordância do questionário aplicado, que apontou a afirmativa "muito satisfeita" com média acima de $90 \%$. Conclusão: a consulta de enfermagem implementada demonstrou ser relevante, corroborando para melhor compreensão do exame, maior qualidade de informação e controle do nível de ansiedade, fato esse evidenciado pela satisfação do usuário com a consulta.

Descritores: Enfermagem no Consultório; Enfermagem Cardiovascular; Satisfação do Paciente; Avaliação.
\end{abstract}

\section{ABSTRACT}

Objective: to assess patient satisfaction with the nursing consultation prior to cardiac catheterization. Method: this quantitative, descriptive study, conducted in the hemodynamics area of a university hospital in Rio de Janeiro State, with the participation of 38 patients who answered the research questionnaire, was approved by the research ethics committee. Results: participants were predominantly male $(20 ; 53 \%)$, over 60 years old $(23 ; 60 \%)$ and arterial hypertension was the most prevalent comorbidity $(33 ; 87 \%)$ and angina, the main reason $(22 ; 58 \%)$. User satisfaction with the nursing consultation was proven by the high rate of agreement with the questionnaire applied: the response "very satisfied" averaged over $90 \%$. Conclusion: the nursing consultation was shown to be important in helping improve patient understanding of the exam, information quality and control of anxiety levels, as evidenced by the users' satisfaction with the consultation.

Descriptors: Office Nursing; Cardiovascular Nursing; Patient Satisfaction; Health evaluation.

\section{RESUMEN}

Objetivo: evaluar la satisfacción de los pacientes en cuanto a la consulta de enfermería antes del procedimiento de cateterismo cardíaco. Método: estudio cuantitativo, descriptivo, realizado en la hemodinámica de un hospital universitario del estado de Río de Janeiro, en el que participaron 38 pacientes que respondieron el cuestionario de investigación, aprobado por el Comité de Ética en Investigación. Resultados: predominó el sexo masculino - 20 (53\%), mayores de 60 años - 23 (60\%). La hipertensión arterial fue la comorbilidad más prevalente - $33(87 \%)$ y la angina fue la razón principal - 22 (58\%). La satisfacción del usuario respecto a la consulta de enfermería quedó comprobada a través del cuestionario aplicado ya que la afirmación 'muy satisfecha' obtuvo un promedio superior al 90\%. Conclusión: la consulta de enfermería implementada se mostró relevante, corroborando para una mejor comprensión del examen, una mayor calidad de información y el control del nivel de ansiedad. Ese hecho se manifiesta por la satisfacción del usuario con la consulta.

Descriptores: Enfermería de Consulta; Enfermería Cardiovascular; Satisfacción del Paciente; Evaluación en Salud.

\section{INTRODUÇÃO}

No atual cenário mundial, as maiores causas de óbitos são as doenças e agravos não transmissíveis (DANT). No Brasil, a mudança no perfil demográfico decorrente dos processos de urbanização e industrialização, alteração do estilo de vida e exposição aos fatores de risco próprios do mundo contemporâneo contribuem para o aumento dessas doenças na população brasileira ${ }^{1}$.

Nesse grupo de doenças, há destaque para as doenças cardiovasculares, câncer, doenças respiratórias crônicas e diabetes, as quais correspondem a 38 milhões de mortes a cada ano. No Brasil, elas representam $72 \%$ das causas de óbitos, expressando um grande problema de saúde pública, sendo as doenças cardiovasculares as primeiras na classificação dos dados de mortalidade ${ }^{2,3}$. 
Dentre as doenças cardiovasculares, a de maior incidência é a Síndrome Coronariana Aguda (SCA) cuja prevalência está, em parte, associada a fatores de risco como tabagismo, hipertensão arterial sistêmica (HAS), diabetes mellitus (DM), dislipidemias, obesidade e sedentarismo. Nos países desenvolvidos, a SCA é responsável por $42 \%$ dos óbitos dentre as DANT e, no Brasil, por mais de $30 \%$ dos óbitos registrados dentre tais doenças ${ }^{4}$.

O conceito de SCA compreende diversas apresentações clínicas decorrentes de isquemia miocárdica, abrangendo a angina instável (AI), o infarto agudo do miocárdio sem supradesnivelamento de ST (IAMSS-ST) e o infarto agudo do miocárdio com supradesnivelamento de ST (IAMCS-ST). Atualmente, a doença cardíaca isquêmica é a principal causa de mortalidade no Brasil e no mundo 5 .

Existem várias estratégias para a avaliação efetiva e segura do paciente com SCA estável, dentre elas a obtenção de eletrocardiograma, dosagem dos marcadores de necrose miocárdica, realização de ecocardiograma transtorácico e de cateterismo cardíaco, sendo esse último, de acordo com a Diretriz de Doença Coronária Estável, o principal exame para o diagnóstico e avaliação das Síndromes Coronarianas Agudas ${ }^{7}$.

O cateterismo cardíaco (ou cineangiocoronariografia) é considerado um exame invasivo, realizado no setor de hemodinâmica por uma equipe de saúde especializada. A equipe de enfermagem que atua neste setor assume vários papéis e realiza diversas ações relacionadas ao procedimento, dentre as quais se destaca a avaliação do paciente antes do cateterismo cardíaco, por meio da consulta de enfermagem. A consulta pré-procedimento permite ao enfermeiro conferir os resultados laboratoriais prévios e condições do paciente que possam impactar no melhor resultado, bem como oferecer orientações sobre o procedimento e cuidados com o local de realização do exame ${ }^{8,9}$.

Em muitos dos indivíduos que se submetem ao exame de cateterismo cardíaco a ansiedade está presente e frequentemente relacionada à ameaça de mudança no estado de saúde, sendo evidenciada por relatos de sensação de angústia, preocupação e expectativa quanto ao resultado do exame diagnóstico e à possibilidade de evolução para outros procedimentos, como a angioplastia e cirurgia cardíaca ${ }^{10}$.

Além disso, a hemodinâmica é um serviço de alta complexidade, extremamente específico e que requer uma equipe de enfermagem atuante, capacitada, eficiente, ágil, atualizada e com processo de trabalho organizado. A consulta de enfermagem previne complicações relacionadas ao procedimento e auxilia o bom funcionamento do setor, como também contribui para que seja prestada uma assistência de qualidade aos pacientes ${ }^{11}$.

Nessa perspectiva, a consulta de enfermagem norteia e colabora para a melhoria do cuidado em saúde, permitindo que o enfermeiro identifique problemas, riscos e as potencialidades do paciente por meio do raciocínio clínico, determinando intervenções e resultados a serem alcançados e dando maior cientificidade a esse conjunto de ações em qualquer nível de saúde, incluindo o setor de exames e procedimentos, como o serviço de hemodinâmica ${ }^{10}$.

Considerando a necessidade contínua de melhoria dos processos nos serviços de saúde, a fim de torná-los mais eficientes e aprimorar a consulta de enfermagem realizada no cenário da hemodinâmica, torna-se importante a avaliação continuada da satisfação do usuário. Assim sendo, é possível identificar as oportunidades de melhoria e traçar intervenções direcionadas ao aperfeiçoamento do processo de cineangiocoronariografia ${ }^{9}$.

A satisfação do usuário pode ser entendida como as expectativas do indivíduo sobre um serviço de saúde oferecido. Remete à avaliação do cuidado recebido, considerando a experiência prévia do paciente com serviços similares. Nesse sentido, a avaliação da satisfação do usuário é adjunta dos aspectos desejáveis no relacionamento entre a equipe de saúde e o paciente e pode contribuir na percepção de quem está recebendo o cuidado, bem como torná-la uma experiência agradável ou ruim ${ }^{12}$.

Entende-se por avaliação a elaboração, negociação e aplicação de critérios explícitos e analisados, utilizando metodologias adequadas, visando conhecer, medir e julgar um contexto, valor ou estado de um objeto, facilitando a melhoria dos processos e desenvolvimento de pessoas ${ }^{13,14}$.

Nessa perspectiva, esse estudo teve como objetivo avaliar a satisfação dos pacientes em relação à consulta de enfermagem prévia ao procedimento de cateterismo cardíaco.

\section{MÉTOdo}

Trata-se de um estudo do tipo descritivo com abordagem quantitativa. O cenário foi o serviço de hemodinâmica de um Hospital Universitário do Estado do Rio de Janeiro. A pesquisa foi realizada entre março de 2018 e setembro de 2018.

No cenário em que a pesquisa se desenvolveu, a consulta de enfermagem é realizada antes do procedimento de cateterismo cardíaco e tem foco na indicação clínica para realização do procedimento, na história pregressa e atual da doença, nos exames diagnósticos e laboratoriais prévios, nos medicamentos que o paciente utiliza, como também nas orientações sobre o procedimento a ser realizado. 
Participaram 38 pacientes selecionados por meio de uma amostra por conveniência. Todos que foram convidados aceitaram participar do estudo. Os critérios de inclusão foram idade igual ou superior a 18 anos; ter realizado a consulta de enfermagem pré-procedimento; possuir no mínimo ensino fundamental incompleto; ser usuário do serviço de saúde público por meio do Sistema Estadual de Regulação (SER); possuir solicitação médica para a realização do cateterismo cardíaco em caráter eletivo.

Foram excluídos os pacientes com barreiras de comunicação e cognição; portadores de deficiência visual, motora e/ou auditiva; pacientes provenientes das unidades de internação da instituição abordada; pacientes submetidos ao exame em caráter de urgência; e pacientes que apresentavam condições clínicas desfavoráveis, como dor, dispneia e fadiga durante a entrevista.

Os dados foram coletados por meio de um questionário elaborado pelas pesquisadoras e composto de duas partes: a primeira com oito variáveis direcionadas à caracterização dos pacientes (sexo, idade, raça, renda mensal, bairro, nível de escolaridade, comorbidades e motivos do exame); e a segunda parte com a escala de Likert contendo 13 indicadores relacionados à avaliação da satisfação do usuário com a consulta de enfermagem, sendo três voltados à dimensão de estrutura (domínio e segurança demonstrados pelo profissional, linguagem utilizada e o ambiente para a realização da consulta) e dez relativos à dimensão de processo (esclarecimentos de dúvidas, orientações sobre o exame, orientações relacionadas aos cuidados pré, trans e pós-procedimento, avaliação do estado de saúde, tempo de duração da consulta, relacionamento entre usuário e profissional, confiança e qualidade da consulta).

O questionário foi aplicado pela pesquisadora principal, que não possui vínculo fixo com o setor, a fim de não caracterizar uma relação de poder diante do paciente e minimizar viés de resposta. No questionário, levou-se em consideração o anonimato do usuário, atribuindo-se uma identificação numérica prévia.

$\mathrm{Na}$ escala do tipo Likert, o pesquisador produz cinco categorias de respostas pontuadas, havendo um ponto neutro no meio da escala. As assertivas a serem empregadas foram produzidas por meio da utilização de verbos tanto na primeira como na terceira pessoa. Essa escala foi selecionada como instrumento em virtude da sua viabilidade de abordagem acerca de interrogações específicas com os pacientes para a conquista dos objetivos da pesquisa. Todavia, consideraram-se apenas as categorias da escala do tipo Likert alterada para a tabulação dos dados $\operatorname{colhidos}^{15}$.

As cinco categorias são: insatisfeito (1 ponto); pouco satisfeito (2 pontos); não sabe ( 3 pontos); satisfeito (4 pontos); e muito satisfeito (5 pontos). Desse modo, atribuíram-se pontuações a cada categoria, em que aquela que corresponde ao menor grau de satisfação possui o menor peso, aumentando a pontuação de forma crescente de acordo com o grau de satisfação do indivíduo em relação ao objeto estudado.

Com o objetivo de simplificar a análise estatística descritiva, os questionários foram codificados e os resultados lançados em uma planilha do Excel ${ }^{\circledR}$.

Os dados abordados foram tabulados e analisados por meio de estatística descritiva simples, medidas de tendência central média, frequência simples e porcentagem.

O presente estudo respeitou as características éticas e as exigências da Resolução no 466, de 12 de dezembro de 2012, do Conselho Nacional de Saúde. A pesquisa foi aprovada em 21 de março de 2018 com parecer de número 2.554.225.

\section{RESULTADOS}

As características sociodemográficas e clínicas dos pacientes submetidos à consulta de enfermagem préprocedimento de cateterismo cardíaco em um hospital universitário do Rio de Janeiro são apresentadas na Tabela 1.

Dos 38 pacientes que participaram da pesquisa, a média de idade foi de 60 anos ( 23 - $60 \%$ com 60 anos ou mais e 3 - 8\% com 30 a 49 anos), a maioria, 20 (53\%), do sexo masculino e a cor parda prevaleceu 18 (47\%). A renda salarial predominante variou entre 1 e 3 salários, 23 (60\%). O ensino médio completo foi a escolaridade mais frequente, $13(34 \%)$.

O fator de risco para doença cardiovascular em evidência foi a hipertensão arterial, 33 (87\%), tendo a angina como motivo para realização do cateterismo, 22 (58\%).

Com relação à satisfação do usuário com a consulta de enfermagem, detalhou-se o grau de concordância dos pacientes envolvidos na pesquisa relacionada com as dimensões de estrutura e processo que foram apresentadas na escala do tipo Likert, como descrito na Tabela 2. 
TABELA 1: Características sociodemográficas e clínicas dos pacientes submetidos à consulta de enfermagem pré-procedimento de cateterismo cardíaco. Rio de Janeiro, RJ, Brasil, 2019.

\begin{tabular}{|c|c|c|}
\hline Características & $\mathbf{n}$ & $\%$ \\
\hline \multicolumn{3}{|l|}{ Sexo } \\
\hline Masculino & 20 & 53 \\
\hline Feminino & 18 & 47 \\
\hline \multicolumn{3}{|l|}{ Idade } \\
\hline 60 ou mais & 23 & 60 \\
\hline 50 a 59 anos & 12 & 32 \\
\hline 30 a 49 anos & 03 & 8 \\
\hline \multicolumn{3}{|l|}{ Raça } \\
\hline Pardo & 18 & 47 \\
\hline Branco & 15 & 39 \\
\hline Negro & 04 & 11 \\
\hline Amarelo & 01 & 3 \\
\hline \multicolumn{3}{|l|}{ Renda Familiar } \\
\hline De 1 a a 3 salários & 23 & 60 \\
\hline Até 1 salário & 09 & 24 \\
\hline De 3 a 5 salários & 03 & 8 \\
\hline De 5 a 7 salários & 03 & 8 \\
\hline \multicolumn{3}{|l|}{ Bairro de Origem } \\
\hline Campo Grande & 03 & 08 \\
\hline Mais 31 bairros & 35 & 92 \\
\hline \multicolumn{3}{|l|}{ Escolaridade } \\
\hline Ensino Médio Completo & 13 & 34 \\
\hline Ensino Médio Incompleto & 10 & 26 \\
\hline Ensino Fundamental & 06 & 16 \\
\hline Ensino Superior Completo & 05 & 13 \\
\hline Ensino Superior Incompleto & 03 & 8 \\
\hline Pós Graduação & 01 & 3 \\
\hline \multicolumn{3}{|l|}{ Fatores de Risco } \\
\hline Hipertensão Arterial & 33 & 87 \\
\hline Diabetes & 14 & 37 \\
\hline Dislipidemias & 13 & 34 \\
\hline Cardiopatias & 07 & 18 \\
\hline Outras comorbidades & 06 & 16 \\
\hline Insuficiência Renal & 02 & 5 \\
\hline Obesidade & 01 & 3 \\
\hline \multicolumn{3}{|l|}{ Motivos do Exame } \\
\hline Angina & 22 & 58 \\
\hline Infarto & 07 & 18 \\
\hline Outros motivos & 09 & 24 \\
\hline
\end{tabular}


TABELA 2: Afirmativas em relação ao grau de satisfação do usuário com a consulta de enfermagem pré procedimento de cateterismo cardíaco. Rio de Janeiro, 2019

\begin{tabular}{|c|c|c|c|c|c|}
\hline Afirmativas & Insatisfeito & $\begin{array}{l}\text { Pouco } \\
\text { Satisfeito }\end{array}$ & Não sabe & Satisfeito & $\begin{array}{l}\text { Muito } \\
\text { Satisfeito }\end{array}$ \\
\hline Processo & $\mathrm{N} / \%$ & $\mathrm{~N} / \%$ & $\mathrm{~N} / \%$ & $\mathrm{~N} / \%$ & $\mathrm{~N} / \%$ \\
\hline $\begin{array}{l}\text { 1. A consulta de enfermagem proporcionou o esclarecimento } \\
\text { de dúvidas sobre o procedimento }\end{array}$ & $0 / 0 \%$ & $1 / 3 \%$ & $0 / 0 \%$ & $2 / 5 \%$ & $35 / 92 \%$ \\
\hline $\begin{array}{l}\text { 2. A enfermeira orientou em relação ao procedimento a ser } \\
\text { realizado }\end{array}$ & $0 / 0 \%$ & $2 / 5 \%$ & $0 / 0 \%$ & $1 / 3 \%$ & $35 / 92 \%$ \\
\hline $\begin{array}{l}\text { 3. A enfermeira orientou sobre os principais cuidados antes do } \\
\text { exame }\end{array}$ & $0 / 0 \%$ & $1 / 3 \%$ & $0 / 0 \%$ & $2 / 5 \%$ & $35 / 92 \%$ \\
\hline $\begin{array}{l}\text { 4. A enfermeira orientou sobre os principais cuidados durante o } \\
\text { exame }\end{array}$ & $1 / 3 \%$ & $3 / 8 \%$ & $0 / 0 \%$ & $1 / 3 \%$ & $33 / 87 \%$ \\
\hline $\begin{array}{l}\text { 5. A enfermeira orientou sobre os principais cuidados após o } \\
\text { exame }\end{array}$ & $5 / 13 \%$ & $5 / 13 \%$ & $1 / 3 \%$ & $1 / 3 \%$ & $26 / 38 \%$ \\
\hline $\begin{array}{l}\text { 6. A enfermeira perguntou sobre o meu estado de saúde para } \\
\text { efetuar o procedimento }\end{array}$ & $0 / 0 \%$ & $1 / 3 \%$ & $0 / 0 \%$ & $0 / 0 \%$ & 37 / 97\% \\
\hline $\begin{array}{l}\text { 7. O tempo de duração da consulta de enfermagem foi } \\
\text { adequado }\end{array}$ & $0 / 0 \%$ & $1 / 3 \%$ & $0 / 0 \%$ & $3 / 8 \%$ & 34 / 89\% \\
\hline $\begin{array}{l}\text { 8. A consulta de enfermagem proporcionou melhoria no } \\
\text { relacionamento do paciente e enfermeiro }\end{array}$ & $0 / 0 \%$ & $0 / 0 \%$ & $0 / 0 \%$ & $3 / 8 \%$ & $35 / 92 \%$ \\
\hline $\begin{array}{l}\text { 9. A consulta de enfermagem aumentou a minha confiança } \\
\text { para realizar o procedimento na unidade }\end{array}$ & $0 / 0 \%$ & $1 / 3 \%$ & $0 / 0 \%$ & $4 / 11 \%$ & 34 / 89\% \\
\hline 10. A consulta de enfermagem realizada é de boa qualidade & $0 / 0 \%$ & $0 / 0 \%$ & $0 / 0 \%$ & $1 / 3 \%$ & $37 / 97 \%$ \\
\hline \multicolumn{6}{|l|}{ Estrutura } \\
\hline $\begin{array}{l}\text { 1. A enfermeira manifestou domínio e segurança a respeito do } \\
\text { procedimento que irei realizar }\end{array}$ & $0 / 0 \%$ & $1 / 3 \%$ & $0 / 0 \%$ & $0 / 0 \%$ & $37 / 97 \%$ \\
\hline $\begin{array}{l}\text { 2. A enfermeira utilizou linguagem simples e de fácil } \\
\text { compreensão para o paciente }\end{array}$ & $0 / 0 \%$ & $0 / 0 \%$ & $0 / 0 \%$ & $1 / 3 \%$ & $37 / 97 \%$ \\
\hline $\begin{array}{l}\text { 3. O ambiente da consulta de enfermagem dispõe de espaço, } \\
\text { ventilação e iluminação adequados }\end{array}$ & $0 / 0 \%$ & $5 / 13 \%$ & $0 / 0 \%$ & $4 / 11 \%$ & $29 / 76 \%$ \\
\hline
\end{tabular}

A afirmativa que apresentou maior frequência de insatisfação foi a relacionada às orientações pós-procedimento, indicada por cinco participantes (13\%). Já o baixo grau de satisfação ("pouco satisfeito") apresentou-se mais frequente na dimensão de estrutura e estava relacionado ao ambiente da consulta de enfermagem adequado, sendo apontado por cinco respondentes (13\%).

As seguintes questões foram as que apresentaram maior grau de satisfação dos pacientes ("muito satisfeito"), nas afirmativas de processo: "A enfermeira perguntou sobre o meu estado de saúde para efetuar o procedimento"; e "A consulta de enfermagem realizada é de boa qualidade", ambas com 97\% de frequência.

Todas as variáveis de estrutura apresentaram frequência acima de $70 \%$, demonstrando alto grau de satisfação dos pacientes ("muito satisfeito").

\section{DISCUSSÃO}

Dentre as características sociodemográficas, observou-se que o sexo masculino prevaleceu com 53\% (20). Pesquisas comprovam esses achados quando discutem que existe um maior risco para evento cardiovascular no homem, visto que o gênero masculino é um fator de risco importante. Porém, 18 (47\%) mulheres foram submetidas ao procedimento de cateterismo cardíaco neste estudo, sendo considerada uma proporção relevante. Isso se deve ao fato de que o gênero feminino, associado à idade avançada e a outros fatores de risco como o diabetes e a dislipidemia, contribui para o aumento do risco de doenças cardiovasculares nessa população. No entanto, as razões fisiológicas para tanto, principalmente, após o climatério, ainda não estão totalmente esclarecidas ${ }^{16,17}$.

A média de idade dos pacientes da pesquisa foi acima de 60 anos. Essas informações vão ao encontro das evidências científicas que referem que a SCA é a mais incidente em população acima de 60 anos, uma vez que o processo de envelhecimento traz consigo muitas consequências para o organismo, sendo a doença crônica não transmissível uma delas. Os dados sociodemográficos e clínicos encontrados se assemelham aos de outro estudo nacional ${ }^{16}$. 
Atualmente, dados apontam a mudança do perfil etário da população brasileira e a relação direta com o aumento das doenças crônicas não transmissíveis, confirmando os achados desta pesquisa. Identificou-se que a maioria da população avaliada apresentou idade próxima aos 60 anos. Estima-se que, a cada ano, 650 mil novos idosos são inseridos na sociedade brasileira, correspondendo a um aumento de quase $7 \%$. Avanços tecnológicos e a melhoria da qualidade de vida são os principais responsáveis por essa transformação ${ }^{1,16}$

A maioria dos pacientes deste estudo apresentou como fator de risco para doença cardiovascular a HAS, sendo relatada em 33 (87\%) dos questionários. Esta é sabidamente um dos fatores de risco mais importantes para a ocorrência de doenças coronarianas, insuficiência cardíaca, doenças cerebrovasculares, fibrilação atrial e insuficiência renal crônica. De acordo com a Organização Mundial da Saúde, a HAS é o principal fator para doenças isquêmicas do coração. Isso demonstra a relevância da realização do rastreamento dos níveis de pressão arterial como medida preventiva em pacientes pré-procedimento de cateterismo cardíaco. Estudos apontam que quando existe associação da HAS com diabetes mellitus, aumenta-se em 2,5 vezes o risco de evento isquêmico ${ }^{18-20}$.

Entre as principais causas para realização do exame nos pacientes incluídos no estudo, a angina destacou-se entre as doenças cardíacas isquêmicas, estando presente em $58 \%$ (22) dos que responderam ao questionário ${ }^{21}$.

No que concerne à satisfação do usuário, identificou-se por meio do grau de concordância dos pacientes, nos indicadores que tiveram avaliação acima de $85 \%$, que 34 (89\%) participantes referiram que a consulta de enfermagem aumentou a confiança para realização do exame, 35 (92\%) afirmaram que esclareceu dúvidas e 37 (97\%) responderam que aprimorou o relacionamento entre profissional e paciente. Observou-se que $97 \%$ dos pacientes manifestaram satisfação com a consulta de enfermagem, reforçando a qualidade e a importância da mesma no esclarecimento de dúvidas e na amenização da expectativa sobre o exame.

A expectativa durante o tempo de espera para realizar o exame, bem como o medo do desconhecido com relação à falta de informação da equipe de saúde, é capaz de gerar ansiedade, o que pode afetar hemodinamicamente os pacientes em decorrência da ativação do sistema nervoso simpático. A pressão arterial, as frequências cardíaca e respiratória, assim como a demanda miocárdica de oxigênio, podem aumentar, interferindo até mesmo no aumento do risco de isquemia e arritmia durante o procedimento ${ }^{22}$.

Entretanto, na pesquisa em tela, constatou-se uma oportunidade de melhoria em relação às orientações sobre os cuidados após o procedimento. Dos 38 pacientes da pesquisa, 28,9\% relataram insatisfação relativa a esse item ou não souberam responder. Esses achados podem ajudar a traçar estratégias a fim de melhorar essa etapa do processo, como intensificar a informação sobre os cuidados com o curativo compressivo, abordar o tempo de permanência no hospital após o exame, orientar sobre as atividades cotidianas que o indivíduo já se encontra apto a desenvolver e possíveis restrições com alimentação, entre outros. A disponibilização de orientações na forma impressa pode ajudar no processo educativo do paciente e dos seus familiares durante a consulta de enfermagem².

A partir da avaliação da satisfação dos pacientes, percebeu-se o quanto a comunicação efetiva e as orientações antes, durante e após o procedimento fazem a diferença no esclarecimento de dúvidas e podem auxiliar na prevenção de eventos adversos, contribuindo para um cuidado seguro e voltado a uma assistência de qualidade. Um paciente bem informado mostra-se menos ansioso e mais seguro ${ }^{23}$.

Estudos apontam que, por meio das orientações, o paciente se sente mais seguro, uma vez que, quando compreende a finalidade e os processos de saúde, torna-se mais fácil o entendimento e a adesão às medidas terapêuticas estabelecidas ${ }^{24,25}$.

A comunicação entre a enfermagem e o paciente contribui para o estabelecimento de um vínculo de confiança e troca de informações, esclarecendo dúvidas e minimizando a ansiedade e insegurança inerentes ao momento que antecede o exame, preservando-o de experiências traumáticas que o ambiente hospitalar proporciona ${ }^{23}$.

Destaca-se que a consulta de enfermagem possibilita ao enfermeiro fornecer as informações necessárias ao paciente, esclarecer dúvidas e obter dados que são relevantes ao procedimento. Com base nesses dados, é possível desenvolver um plano de ação visando minimizar os riscos oferecidos tanto pelo uso de contraste iodado quanto pela exposição ionizante, além de melhorar a relação entre paciente, equipe de enfermagem e família ${ }^{26,27}$

Na dimensão da qualidade da consulta de enfermagem, identificou-se o quanto esta é significativa no processo de realização do procedimento de cateterismo cardíaco, uma vez que o grau de satisfação dos pacientes foi positivo e elevado, reafirmando a importância e a relevância do enfermeiro no âmbito da hemodinâmica, ressaltando o seu papel e a sua contribuição na construção de um cuidado seguro e na assistência de qualidade, tendo como resultado um procedimento seguro e um usuário satisfeito.

Este estudo teve como limitações o fato de a pesquisa ser realizada em apenas um centro hospitalar, proporcionando, assim, um número reduzido de pacientes. Há de se considerar também o fato de que, por vezes, o 
indivíduo pode se sentir envergonhado para expressar suas insatisfações, temendo retaliações caso necessite retornar ao serviço de saúde. Recomenda-se tornar rotineira a avaliação da satisfação do usuário, de maneira formal, por meio de questionários ou contatos telefônicos, a fim de manter em constante observação a qualidade da consulta ${ }^{8}$.

Estima-se que esta pesquisa possa contribuir de forma significativa para a valorização do papel do enfermeiro no que concerne ao preparo do paciente para o procedimento de cateterismo cardíaco, ratificando como a consulta de enfermagem antes do exame pode amenizar as expectativas do paciente que vai ser submetido ao procedimento ${ }^{29}$, bem como contribuir para a identificação de indicadores clínicos que possam impactar no melhor resultado e menor risco para o usuário.

Sob a perspectiva do estudo, ressalta-se que são escassos aqueles que abordam a consulta de enfermagem no serviço de hemodinâmica. Nesse sentido, pretende-se colaborar com a formação científica a respeito da temática, possibilitando o aprofundamento do objeto em questão, ampliando espaços de comunicação e interação entre os pacientes e profissionais que atuam nos serviços de hemodinâmica.

\section{CONCLUSÃO}

Evidenciou-se que a prevalência da idade dos pacientes que realizam o cateterismo cardíaco foi acima de 60 anos, com predomínio do sexo masculino, o que condiz com a média apontada nos estudos, diretamente relacionada a fatores preexistentes.

As seguintes afirmativas foram as que apresentaram maior grau de satisfação: “A enfermeira perguntou sobre o meu estado de saúde para efetuar o procedimento"; "A enfermeira manifestou domínio e segurança a respeito do procedimento que irei realizar"; e "A enfermeira utilizou linguagem simples e de fácil compreensão para o paciente". Todas com 97\% de satisfação.

A questão que apresentou maior grau de insatisfação foi a relacionada às orientações dos principais cuidados após o exame. Entende-se que identificar os pontos de fragilidade da consulta ajuda a traçar estratégias para melhorar etapas específicas do processo.

Apesar dos resultados positivos em relação ao grau de satisfação do paciente, percebe-se que o aprimoramento dos pontos negativos deve ser levado em consideração para que haja melhoria da consulta de enfermagem no serviço de hemodinâmica em que foi realizado o estudo. Destaca-se que a reavaliação e a melhoria contínua dos processos devem fazer parte da rotina dos serviços que se propõem a desenvolver um cuidado seguro e com qualidade.

A avaliação da consulta de enfermagem implementada demonstrou ser de grande relevância para atuação do enfermeiro no serviço de hemodinâmica, corroborando para melhor compreensão do paciente sobre o exame, maior qualidade de informações direcionadas aos usuários, maior controle das expectativas por parte do paciente, além de apontar lacunas que precisam ser trabalhadas para o aprimoramento da consulta de enfermagem no cenário estudado, principalmente no que tange às orientações pós-procedimento. Avaliar a satisfação do paciente é uma técnica que permite ao enfermeiro desenvolver o processo educativo, valorizar as dúvidas do paciente e contribuir para a melhoria das condições de saúde.

\section{REFERÊNCIAS}

1. Calais NVM, Teixeira ND, Bonin KM, Mirely S. Consumption and social and demographic profile of the different household arrangements of the elderly in Brazil: analysis from the Study on Family Budgets Rev. bras. geriatr. gerontol. 2017 [cited 2019 Jan 10]; 20(5):607-17. DOI: http://dx.doi.org/10.1590/1981-22562017020.170047.

2. Andrade KBS, Pinheiro APB, Bessa ATT, Paes GO, Stipp MAC. Evaluation of waiting times for initial electrocardiogram in patients with Acute Coronary Syndrome. Rev. enferm. UERJ. 2015 [cited 2019 Jan 10]; 23(4):443-8. DOI: http://dx.doi.org/10.12957/reuerj.2015.18332.

3. Toso A, Leoncini M, Maioli M, Bellandi F. Pharmacologic Prophylaxis of Contrast-Induced Nephropathy. Interventional Cardiology Clinics. 2020 [cited 2019 Jan 10]; 9(3):369-83. DOI: https://doi.org/10.1016/j.iccl.2020.02.006.

4. Freitas EO, Nogueira RS, Stekel LMC, Bublitz S, Kirchhof R, Guido LA. Profile of patients with coronary artery disease undergoing cardiac catheterization Rev. enferm. UFSM. 2013 [cited 2019 Jan 10]; 3(Esp.):679-88. DOI: https://doi.org/10.5902/2179769211124.

5. Nicolau JC, Timerman A, Marin-Neto JA, Piegas LS, Barbosa CJDG, Franci A. Sociedade Brasileira de Cardiologia. Diretrizes da Sociedade Brasileira de Cardiologia sobre Angina Instável e Infarto Agudo do Miocárdio sem Supradesnível do Segmento ST. Arq. Bras. Cardiol. 2014 [cited 2019 Jan 10]; 102(3Supl.1):1-61. DOI: https://doi.org/10.5935/abc.2014S001.

6. Précoma DB, Oliveira GMM, Simão AF, Dutra, OP, Coelho OR, Izar COM et al. Updated Cardiovascular Prevention Guideline of the Brazilian Society of Cardiology - 2019. Arq. bras. cardiol. 2019[cited 2019 Jan 10]; 113(4):787-891. DOI: https://doi.org/10.5935/abc.20190204. 
7. Jacinto LAT, Santos AS, Diniz MA, Silva LC, Pedrosa FSS, Arduini JB. Coronary artery disease and family support in older adults. Rev Enferm UERJ. 2014 [cited 2019 Jan 10]; 22(6):771-7. DOI: https://doi.org/10.12957/reuerj.2014.15664.

8. Teixeira TRF e Braga EM. Dissertação de Mestrado: Cateterismo Cardíaco da Compreensão do Usuário ao Planejamento das Orientações de Enfermagem. Botucatu-São Paulo; 2016.

9. Capetini, AC, Camacho, ACLF. Nursing care in the hemodynamics service in interventional cardiology: integrative review. Research, Society and Development. 2020 [cited 2019 Jan 10]; 9(7):e284974200. DOI: http://dx.doi.org/10.33448/rsdv9i7.4200.

10. Silva MAS, Marques PTV, Castro DFA, Padula MPC, Yano KT, Coimbra ALL, et al. Relation between orientation, anxiety and pain in cardiac catheterization. Arq. Med. Hosp. Fac. Cienc. Med. 2016 [cited 2019 Jan 10]; 61(1):28-34. Available from: http://arquivosmedicos.fcmsantacasasp.edu.br/index.php/AMSCSP/article/view/130.

11. Rodrigues MGJ, Silva R, Dias MG, Paraíso AF. Nursing process in patients submitted to coronary percutaneous transluminary angioplasty. REAS. 2019 [cited 2020 Jan 11]; 23(1):e284. Available from: https://acervomais.com.br/index.php/saude/article/view/284 12.

12. Inchauspe JAF, Moura GMS. Applicability of the results of a user satisfaction survey by nursing. Acta Paul. Enferm. 2015 [cited 2016 Jan 14]; 28(2):177-82. DOI: https://doi.org/10.1590/1982-0194201500030.

13. Donabedian A. La calidad de la atención médica. Definición y métodos de evaluación. México: La Prensa Médica Mexicana; 1984.

14. Paes NA, Silva CS, Figueiredo TMRM. Satisfaction of hypertensive users with primary health care services in Brazil: a validation study. Rev. Panam. Salud pública. 2014 [cited 2019 Set 9]; 36(2):87-93. Available from: http://www.scielosp.org/pdf/rpsp/v36n2/03.pdf.

15. Dalmoro M; Vieira, KM. Dilemmas of the type likert scales construction: does the number of items and the disposition influence results?. Rev. Gestão Organizacional. 2013 [cited 2020 Jan 11]; 6(3):161-74. Available from: http://www.spell.org.br/documentos/ver/31731/dilemmas-of-the-type-likert-scales-construction---/i/en.

16. Marotto KV, Rocha RG, Naves CBO, Almeida LF, Marta CB, Oliveira CSR. Clinical and epidemiological profile of patients undergoing cardiac catheterization at a university hospital in Rio de Janeiro. Rev. Fun. Care Online. 2019 [cited 2020 Jan 11]; 11(4):894-899. DOI: http://dx.doi.org/10.9789/2175-5361.2019.v11i4.894-899.

17. Arruda GO, Marcon SS. Health risk behaviors of men from the southern Brazil. Texto \& contexto enferm. 2018 [cited 2020 Jan 11]; 27(2): e2640014. DOI: https://doi.org/10.1590/0104-070720180002640014.

18. Khorrami Z, Rezapour M, Koorosh E, Yarahmadi S, Khodakarim S et al. The patterns of Non-communicable disease Multimorbidity in Iran: a multilevel analysis. Sci. Rep. 2020 [cited 2020 Jan 11]; 10(1):30-34. DOI: https://doi.org/10.1038/s41598-020-59668-y.

19. Toshima T, Tomoharu Y, Yukiko KF, Takeshi K, Yoshiya S, Mano Y et al. Risk factors for the metabolic syndrome components of hypertension, diabetes mellitus, and dyslipidemia after living donor liver transplantation. HPB Jornal. 2020 [cited 2020 Jan 11]; 24(1):1-10. DOI: https://doi.org/10.1016/j.hpb.2019.08.008.

20. Flor LS, Campos MR. The prevalence of diabetes mellitus and its associated factors in the Brazilian adult population: evidence from a population-based survey. Rev. bras. Epidemiologia. 2017 [cited 2018 Jun 02]; 20(1):16-29. DOI: http://dx.doi.org/10.1590/1980-5497201700010002.

21. Braga ALS, Valle WAC, Machado MED, Souza DF, Andrade M, Aloi AP. Nursing consultation - a restructuring strategy of the program hiperdia consulta de enfermería - una estrategia de reestructuración del programa hiperdia. Rev. enferm. UFPE on line. 2015 [cited 2020 Jan 12]; 9(6):8155-64. Available from: https://periodicos.ufpe.br/revistas/revistaenfermagem/article/download/10573/11514.

22. Adib HM, Moradi T, Mohseni R. Effects of a multimodal preparation package on vital signs of patients waiting for coronary angiography. Nurs Midwifery Stud. 2014 [cited 2020 Jul 01]; 3(1):e17518. DOI: http://dx.doi.org/10.17795/nmsjournal17518.

23. Moreira FTLS, Macêdo CRC, Albuquerque GA, Meneses RO. Effective communication strategies for managing disruptive behaviors and promoting patient safety. Rev. Gaúcha Enferm. 2019 [cited 2020 Jul 01]; 40(esp): e20180308. DOI: http://dx.doi.org/10.1590/1983-1447.2019.20180308.

24. Emiliano MS, Lindolpho MC, Valente GSC. Perception of nursing consultation by elderly people and their caregivers. Rev. enferm. UFPE on line.2017 [cited 2020 Jan 11]; 11(5):1791-7. Available from: https://periodicos.ufpe.br/revistas/revistaenfermagem/article/download/23325/18913.

25. Silva VFD, Lohmann PM, Pissaia LF, Costa AEK. Cardiac catheterization: the interview in front of the patient's wishes. Research, Society and Development. 2018 [cited 2020 Jan 11]; 7(12):e12712500. DOI: https://doi.org/10.33448/rsd-v7i12.500.

26. Moreira MLAP, Mizuno E, Meireles GCX. Pre-catheterism nursing consultation and percutaneous coronary interventions. Rev. enferm. UFPE online. 2017 [cited 2020 Jan 12]; 11 (Supl. 6): 2548-56. Available from: https://periodicos.ufpe.br/revistas/revistaenfermagem/article/download/23423/19106.

27. Siqueira ASE, Siqueira-Filho AG, Land MGP. Analysis of the economic impact of cardiovascular diseases in the last five years in Brazil Arq. Bras. Cardiol. 2017 [cited 2020 Jan 14]; 109(1):39-46. DOI: https://doi.org/10.5935/abc.20170068.

28. Arruda CAM, Bosi MLM. User's satisfaction of primary health care: a qualitative study in the Northeast of Brazil. Interface (Botucatu). 2016 [cited 2020 Jan 12];21 (61) 321-32. DOI: http://dx.doi.org/10.1590/1807-57622015.0479.

29. Andrade FM, Castro JFL, Silva AV. Perception of pregnant women on the consultation medical and nursing in low-risk prenatal. R. Enferm. Cent. O. Min. 2016 [cited 2020 Jan 12]; 6(3):2377-88 DOI: https://doi.org/10.19175/recom.v6i3.1015. 